\title{
Control of Modular Multilevel Converters under Singular Unbalanced Voltage Conditions with Equal Positive and Negative Sequence Components
}

\author{
Eduardo Prieto-Araujo, Member, IEEE, Adrià Junyent-Ferré, Member, IEEE, Gerard Clariana-Colet, \\ and Oriol Gomis-Bellmunt, Senior Member, IEEE
}

\begin{abstract}
This article focuses on the control of Modular Multilevel Converters (MMC) for High Voltage DC (HVDC) applications during unbalanced $\mathrm{AC}$ grid voltage sags where positive and negative sequence voltages are equal. The control scheme is based on six arm energy regulators, six independent current controllers and two reference calculation stages that convert the power references into grid and inner current references. Conventional inner AC currents reference calculation fails if the amplitude of the positive and the negative sequence $\mathrm{AC}$ grid voltages are equal, a state which is referred to in this paper as singular voltage condition. This article discusses the types of network faults that cause this condition and proposes three different solutions to operate the converter in such scenarios. The adequacy of the proposed solutions is validated through simulations considering each of the problematic fault scenarios.
\end{abstract}

Index Terms-HVDC transmission, modular multilevel converters, voltage-source converter, unbalanced operation.

\section{INTRODUCTION}

$\mathbf{T}$ WO High Voltage DC (HVDC) power transmission technologies are available today: Line-Commutated Converters (LCC) based on thyristors and Voltage Source Converters (VSC) based on Insulated-Gate Bipolar Transistors (IGBT). VSC technology is more suitable for offshore transmission systems as it can black-start, it has enhanced independent active and reactive power control, and most importantly it results in marine substations with smaller footprint [1], [2].

For a VSC-HVDC transmission, the Modular Multilevel Converter (MMC) [3] is the preferred topology. MMCs combine a large number of individually-controlled sub-modules (cells) (see Fig. 1). They can generate high quality output voltage waveforms while maintaining a reduced switching frequency for each sub-module, therefore reducing the switching

E. Prieto-Araujo and O. Gomis-Bellmunt are with the Departament d'Enginyeria Elèctrica, Centre d'Innovació Tecnològica en Convertidors Estàtics i Accionaments (CITCEA-UPC), Universitat Politècnica de Catalunya, ETS d'Enginyeria Industrial de Barcelona, Pl. 2. 08028, Barcelona, Spain. (e-mail: eduardo.prieto-araujo@citcea.upc.edu).

O. Gomis-Bellmunt is also with the Power Electronics and Electric Power Grids Department, Catalonia Institute for Energy Research (IREC), Jardins de les Dones de Negre, 1, 2a pl., 08930, St. Adrià de Besòs, Barcelona, Spain.

A. Junyent-Ferré is with the Department of Electrical and Electronic Engineering, Imperial College London, South Kensington Campus, London SW7 2AZ, UK

G. Clariana-Colet is with Ing. Karl und Albert KRUCH GmbH (KRUCH), Pfarrgasse, 87, 1230, Vienna, Austria.

This work has been funded by the Spanish Ministry of Economy and Competitiveness under the projects ENE2013-47296 and ENE2015-67048-C4-1-R. This research was co-financed by the European Regional Development Fund (ERDF). losses. Based on this concept, different manufacturers have proposed their specific solutions [4]-[6].

The control of the MMC has been addressed in the literature [7]-[11]. The operation of the MMC during unbalanced AC [12], [13] and DC [12], [14] grid conditions has also been studied. The specific case of the singular voltage condition, where positive and negative sequence of the AC grid voltages are equal, is highly problematic as it may result in singularities in the calculation of current references. This situation has been studied in the past for two-level VSCs [15] where the resulting low-frequency power ripple going into the DC bus was a concern. A similar problem arises in the MMC for both the AC grid and inner current reference calculation stages [13], [16]. The discussion introduced in [15] for AC grid currents reference calculation is also valid for MMCs. Regarding the inner current references, a solution based on applying offset voltage was presented in [16] for double line faults. In the present paper, three alternative methodologies that enable the operation of the converter under all possible faults that cause the singular voltage condition, are proposed. The first methodology is based on disabling the problematic elements of the arm energy balancing controllers upon detection of the singular voltage condition. The two other methods are based on solving the current reference calculation problem using linear algebra tools for incompatible systems. In order to validate these methods, a detailed analysis of the different types of voltage sags that result in singular voltage conditions is developed. Simulations are carried out to demonstrate the performance achieved using the different methods.

\section{CONVERTER DESCRIPTION AND CONTROL}

Fig. 1 shows the simplified diagram of a three-phase MMC. The converter has three phase units (known as legs) with two stacks of sub-modules (SM) in each, known as upper and lower arms. Each arm has $N_{\text {arm }} \mathrm{SMs}$, which in the basic MMC present a half-bridge topology [3]. Each SM can be either inserted or by-passed, allowing the arm to behave as a positive controllable voltage source [8]. The voltage applied by the arms is used to control their current, which in turn is used to transfer power and achieve internal energy balance in the converter. 


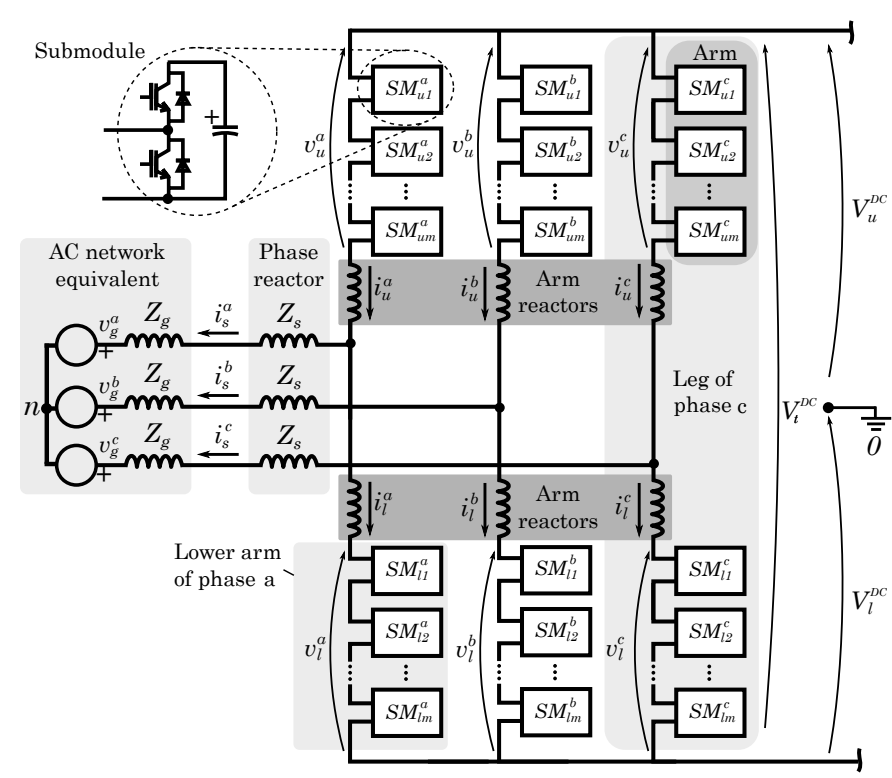

Fig. 1. Complete scheme of an MMC converter.

\section{A. System variables}

The basic relevant variables of the converter ${ }^{1}$ are shown in Fig. 1: $v_{g}^{j}$ is the grid voltage, $v_{u}^{j}$ and $v_{l}^{j}$ are the upper and lower arm voltages respectively and $V_{u}^{D C}$ and $V_{l}^{D C}$ are the voltages of the upper and the lower poles of the HVDC link. Also, another two variables, that do not appear in Fig. 1, but play an important role in the energy balancing of the converter are the sums of the SM capacitor voltages of the upper and the lower arms $v_{u-s}^{j}$ and $v_{l-s}^{j}$, respectively. The relevant currents are the grid current $i_{s}^{j}$ and the upper $i_{u}^{j}$ and lower $i_{l}^{j}$ converter arm currents. Regarding the converter circuit impedances, $R_{a}$ and $L_{a}$ are the equivalent arm resistance and the inductance of the arm reactors respectively, $R_{s}$ and $L_{s}$ are the resistance and inductance of the phase inductors ${ }^{2}$ and $R_{g}$ and $L_{g}$ correspond to the equivalent impedances of the $\mathrm{AC}$ grid. For a better understanding of the converter, a typical change of variable is applied to the MMC equations [12]:

$$
\left\{\begin{array} { l } 
{ v _ { \text { diff } } ^ { j } \triangleq \frac { 1 } { 2 } ( - v _ { u } ^ { j } + v _ { l } ^ { j } ) } \\
{ v _ { \text { sum } } ^ { j } \triangleq v _ { u } ^ { j } + v _ { l } ^ { j } } \\
{ i _ { \text { sum } } ^ { j } \triangleq \frac { 1 } { 2 } ( i _ { u } ^ { j } + i _ { l } ^ { j } ) }
\end{array} \quad \text { and } \quad \left\{\begin{array}{l}
v_{u}^{j}=-v_{\text {diff }}^{j}+\frac{1}{2} v_{\text {sum }}^{j} \\
v_{l}^{j}=v_{\text {diff }}^{j}+\frac{1}{2} v_{\text {sum }}^{j} \\
i_{u}^{j}=\frac{1}{2} i_{s}^{j}+i_{\text {sum }}^{j} \\
i_{l}^{j}=-\frac{1}{2} i_{s}^{j}+i_{\text {sum }}^{j}
\end{array}\right.\right.
$$

where $v_{d i f f}^{j}$ and $v_{\text {sum }}^{j}$ are the differential and the additive voltages applied by the converter, and $i_{\text {sum }}^{j}$ is the additive (inner) current flowing through the converter.

\section{B. Converter control system}

The control scheme employed (see Fig. 2) follows the design procedure introduced in [12]. $P_{g}^{*}$ and $Q_{g}^{*}$ are the $\mathrm{AC}$

\footnotetext{
${ }^{1}$ Superscript $j$ indicates a generic converter phase $(j=a, b, c)$

${ }^{2}$ In some cases the leakage inductance of a grid-interface transformer may be used as the phase inductor.
}

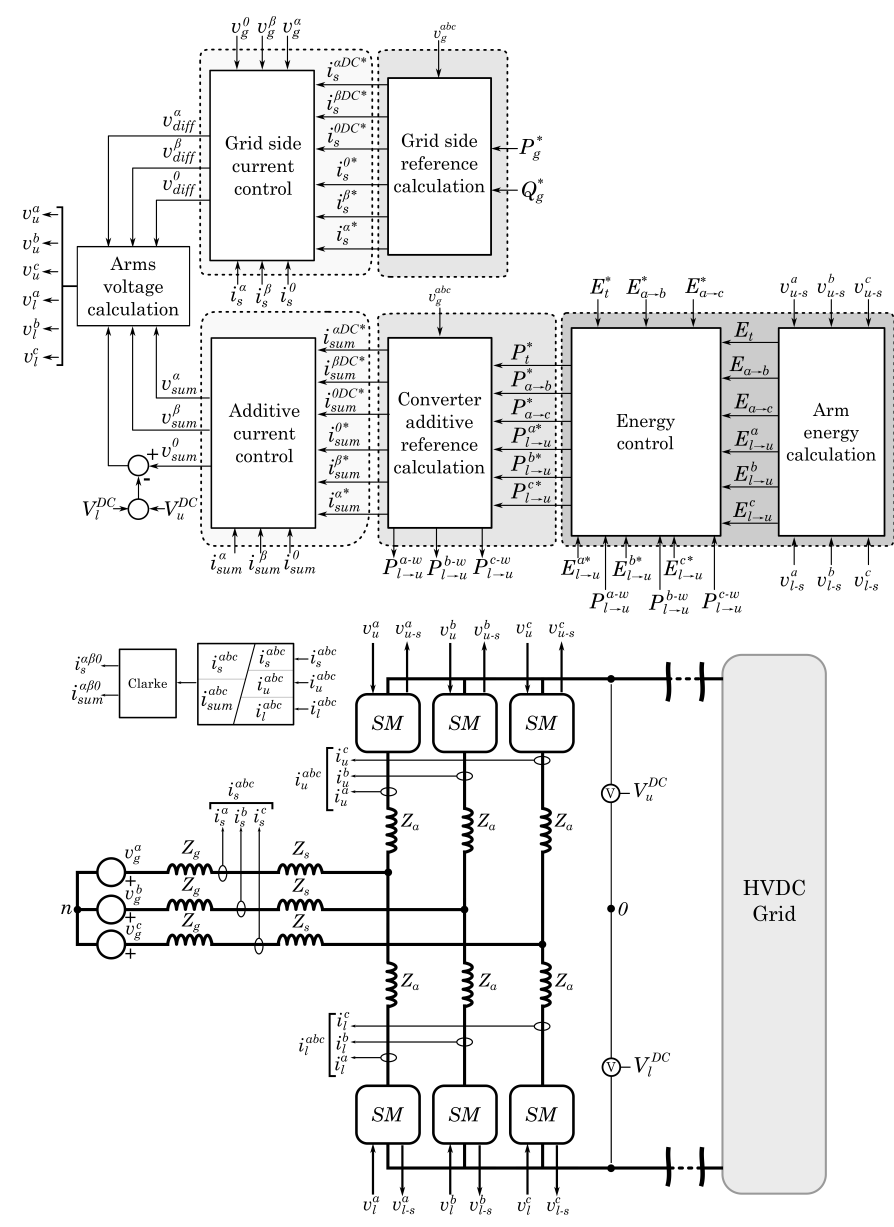

Fig. 2. Control structure of the MMC converter.

grid active and reactive power references. The corresponding AC output current reference $i_{s}^{\alpha \beta 0 *}$ is obtained through a reference calculation structure which takes into account unbalanced voltage situations [15] while its DC component $i_{s}^{\alpha \beta 0 D C *}$ is set to zero to prevent $\mathrm{DC}$ current flowing through the $\mathrm{AC}$ grid, for instance to avoid transformer saturation. In order to balance the internal energy of the converter, six separated energy control loops are required. Specifically, the controlled energy variables are the total energy of the converter $E_{t}$ the energy differences between the converter legs $E_{a \rightarrow b}$ and $E_{a \rightarrow c}$, and the energy difference between the upper and lower arms of the MMC $E_{l \rightarrow u}^{j}$. The output of the energy regulators are the power references $P_{t}^{*}, P_{a \rightarrow b}^{*}, P_{a \rightarrow c}^{*}$ and $P_{l \rightarrow u}^{j *}$. These are fed to a reference calculation stage in order to obtain the additive $\mathrm{AC} i_{\text {sum }}^{\alpha \beta 0 *}$ and DC current references $i_{\text {sum }}^{\alpha \beta 0 D C *}$, which are tracked using the current controllers. All current controllers are designed to track current references in the stationary frame containing $\mathrm{AC}$ and $\mathrm{DC}$ components.

\section{Energy balance control between upper and lower arms}

As mentioned in the previous section, three different regulators are in charge of balancing the energy difference between the upper and the lower arms within a phase unit $E_{l \rightarrow u}^{j}$. Fig. 3 shows a possible implementation of these controllers which consists of three different PI compensators that can be 


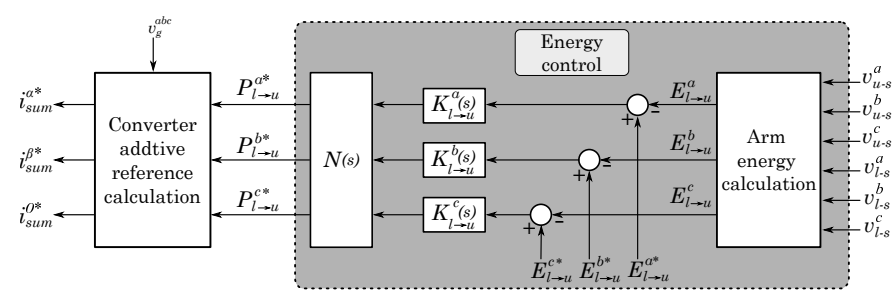

Fig. 3. Upper-lower arms energy balancing control structure

designed to reject disturbances that may appear during the converter operation. Apart from the PI controllers, the scheme also includes a notch filter $N(s)$ to block sending line and double-line frequency magnitudes to the reference calculation stage, as the additive current references are obtained using only the average power values. Note that, the additive reference calculation stage could be combined with other energy regulator structures. For certain voltage conditions, the AC additive current reference calculation is not able to extract valid current references, affecting the energy balancing between the upper and lower arms, as it is detailed in the next section.

\section{REFERENCE CALCULATION PROBLEM}

Table I summarizes the different current components flowing through the converter [12] and their uses within the control structure. The reference values of all components of the current are calculated from the power references given the instantaneous measured AC and DC grid voltages. A variation of the AC grid voltage causes the AC components to change, whereas a variation of the DC grid voltage causes the DC components to change.

The choice of the AC grid current reference during an unbalanced voltage sag has been discussed in the past [15] and the most common approach to date is to set its negative sequence to zero and to export active and reactive power using positive sequence current only. In such a scenario, the active power exchanged between each leg of the converter and the AC grid is different, resulting in a sustained drift of their energy that must be compensated using additive DC current.

On the other hand, the additive AC current is normally used to control the differences between the energy of the upper arms and the energy of the lower arms, which may suffer from deviations during transients. The output voltage of the converter is close to the grid voltage, therefore the power exchanged between upper and lower arms per phase $P_{l \rightarrow u}^{j}$, eliminating the oscillatory terms, can be expressed as [13]

$$
\underbrace{\left[\begin{array}{c}
P_{1} \\
P_{2} \\
P_{3}
\end{array}\right]}_{P}=\underbrace{\left[\begin{array}{ccc}
V_{g}^{+} & 0 & V_{g}^{-} \cos \psi \\
0 & V_{g}^{+} & -V_{g}^{-} \sin \psi \\
V_{g}^{-} \cos \psi-V_{g}^{-} \sin \psi & V_{g}^{+}
\end{array}\right]}_{\mathbf{X}} \underbrace{\left[\begin{array}{c}
I_{\text {sum }}^{-} \cos \alpha \\
-I_{\text {sum }}^{-} \sin \alpha \\
I_{\text {sum }}^{+}
\end{array}\right]}_{I}
$$

where $V_{g}^{+}$is the RMS positive sequence voltage, $V_{g}^{-}$is the RMS negative sequence voltage, and $\psi$ is the angle between the positive and the negative sequence voltage. Regarding the currents, $I_{\text {sum }}^{+}$is the RMS positive sequence component of the additive AC current (which is chosen to be in phase with the positive sequence voltage), $I_{\text {sum }}^{-}$is the RMS negative sequence component of the additive $\mathrm{AC}$ current, and $\alpha$ is the angle between the negative sequence current and the positive sequence voltage, [12]. The power references $P_{1}, P_{2}, P_{3}$ are obtained as a combination of power exchanged between upper and lower arms of each leg, $P_{l \rightarrow u}^{j}$ :

$$
\begin{array}{r}
P_{1} \triangleq \frac{1}{3}\left(-P_{l \rightarrow u}^{c}-P_{l \rightarrow u}^{b}+2 P_{l \rightarrow u}^{a}\right) \\
P_{2} \triangleq \frac{1}{3}\left(\sqrt{3} P_{l \rightarrow u}^{c}-\sqrt{3} P_{l \rightarrow u}^{b}\right) \\
P_{3} \triangleq \frac{1}{3}\left(P_{l \rightarrow u}^{a}+P_{l \rightarrow u}^{b}+P_{l \rightarrow u}^{c}\right)
\end{array}
$$

This system of equations can be inverted in order to obtain the additive $\mathrm{AC}$ current references as a function of the power references given by the energy controllers that maintain the energy balance between upper and lower arms (see Fig. 2):

$$
\begin{gathered}
\underbrace{\left[\begin{array}{c}
I_{\text {sum }}^{-} \cos \alpha \\
-I_{\text {sum }}^{-} \sin \alpha \\
I_{\text {sum }}^{+}
\end{array}\right]}_{I}=\underbrace{\frac{1}{\left(V_{g}^{+}\right)^{2}-\left(V_{g}^{-}\right)^{2}} \cdot\left[\begin{array}{lll}
M_{11} & M_{12} & M_{13} \\
M_{21} & M_{22} & M_{23} \\
M_{31} & M_{32} & M_{33}
\end{array}\right]}_{\mathbf{M}} \underbrace{\left[\begin{array}{c}
P_{1} \\
P_{2} \\
P_{3}
\end{array}\right]}_{P} \\
M_{11}=\frac{2\left(V_{g}^{+}\right)^{2}+(\cos (2 \psi)-1)\left(V_{g}^{-}\right)^{2}}{2 V_{g}^{+}} \\
M_{22}=\frac{2\left(V_{g}^{+}\right)^{2}+(-\cos (2 \psi)-1)\left(V_{g}^{-}\right)^{2}}{2 V_{g}^{+}} \\
M_{12}=M_{21}=-\frac{\sin (2 \psi)\left(V_{g}^{-}\right)^{2}}{2 V_{g}^{+}} ; \quad M_{33}=V_{g}^{+} \\
M_{13}=M_{31}=-V_{g}^{-} \cos \psi ; \quad M_{23}=M_{32}=V_{g}^{-} \sin \psi
\end{gathered}
$$

The solution has a discontinuity when $V_{g}^{+}=V_{g}^{-}$(singular voltage condition), resulting in infinite current being requested when positive and negative sequence voltages are very close in magnitude. This singularity causes the additive current references to saturate, which compromises the performance

\begin{tabular}{|c|c|c|c|}
\hline Comp. & Freq. & Comp. & Use \\
\hline \multirow{4}{*}{$I_{s}$} & \multirow[t]{2}{*}{$\mathrm{AC}$} &,+- & $\begin{array}{l}\text { Active and reactive current to the } \mathrm{AC} \text { grid } \\
\text { (continuous). }\end{array}$ \\
\hline & & 0 & Equal to 0 due to three-wire connection. \\
\hline & \multirow[t]{2}{*}{ DC } & $\alpha, \beta$ & $\begin{array}{l}\text { Controlled to zero to prevent DC current } \\
\text { flowing through the AC grid. }\end{array}$ \\
\hline & & 0 & Equal to 0 due to three-wire connection. \\
\hline \multirow{4}{*}{$I_{\text {sum }}$} & \multirow[t]{2}{*}{$\mathrm{AC}$} &,+- & $\begin{array}{l}\text { Internal power exchange between upper } \\
\text { and lower arms (transient). }\end{array}$ \\
\hline & & 0 & $\begin{array}{l}\text { Controlled to zero to avoid AC distortion } \\
\text { in the DC grid. }\end{array}$ \\
\hline & \multirow[t]{2}{*}{ DC } & $\alpha, \beta$ & $\begin{array}{l}\text { Internal power exchange between the legs } \\
\text { of the converter (continuous/transient). }\end{array}$ \\
\hline & & 0 & $\begin{array}{l}\text { Power flowing onto the DC grid } \\
\text { (continuous). }\end{array}$ \\
\hline
\end{tabular}
of the energy-balancing controllers.

This reference calculation problem could also appear for other control strategies different than the control scheme shown in Fig. 2, provided that the control method uses AC additive currents to balance the energy stored in the upper and lower arms.

TABLE I

CONVERTER CURRENT COMPONENTS AND THEIR USES 


\section{MODIFICATION OF THE ADDITIVE CURRENT CALCULATION TECHNIQUE}

This section discusses three different methods that can be used to overcome the problem presented in the previous part.

1) Method 1 - Temporary disabling the upper to lower arms energy balance control: Unbalanced AC voltage sags do not cause sustained drift between the energy of the upper arms and the lower arms of the converter. However, stepwise deviations may be caused by transients. The normal approach to control these deviations (see (3)) would require very large additive AC current under voltage imbalances close to the singular voltage condition. Thus, a possible solution is to disable the energy controller that balances the differences between the energy of the upper and the lower arms during the voltage sag. The main drawback of this method is that the energy deviation may build up during very long fault clearance times and will not be reduced until the fault is cleared, and the energy regulator is activated again.

2) Method 2 - Kernel-based approach: The second approach is based on the idea of avoiding the use of current that does not contribute to power exchange. Such current can be found by studying the kernel of matrix $\mathbf{X}$ in (1), which loses rank under singular voltage conditions. A vector $v$ is said to belong to the kernel of $\mathbf{X}$ if $\mathbf{X} \cdot v=0$. Such vector can be easily found using classic linear algebra techniques and yields

$$
v=\left[\begin{array}{lll}
\frac{V_{g}^{-} \cos \psi}{\sqrt{V_{g}^{+2}+V_{g}^{-2}}} & \frac{-V_{g}^{-} \sin \psi}{\sqrt{V_{g}^{+2}+V_{g}^{-2}}} & \frac{-V_{g}^{+}}{\sqrt{V_{g}^{+2}+V_{g}^{-2}}}
\end{array}\right]^{T}
$$

The vector only belongs to the kernel $\mathbf{X}$, when $\mathbf{X}$ loses rank, this can be easily proven by multiplying $v$ by $\mathbf{X}$

$$
\mathbf{X} \cdot v=\left[\begin{array}{lll}
0 & 0 & -\frac{V_{g}^{+2}-V_{g}^{-2}}{\sqrt{V_{g}^{+2}+V_{g}^{-2}}}
\end{array}\right]^{T}
$$

where it can be seen that a vector of current $v$ does not produce any sustained power exchange under the condition of $V_{g}^{+}=V_{g}^{-}$. A linear transformation matrix, $\mathbf{S}$ can be used to express the additive current in a new base where the third component corresponds to the direction that does not produce power exchange under singular voltage conditions

$$
\mathbf{S}=\left[\begin{array}{ccc}
1 & 0 & \frac{V_{g}^{-} \cos \psi}{\sqrt{V_{g}^{+2}+V_{g}^{-2}}} \\
0 & 1 & \frac{-V_{g}^{-} \sin \psi}{\sqrt{V_{g}^{+2}+V_{g}^{-2}}} \\
0 & 0 & \frac{-V_{g}^{+}}{\sqrt{V_{g}^{+2}+V_{g}^{-2}}}
\end{array}\right]
$$

Applying this transformation to (1) leads to

$$
P=\mathbf{X} \cdot I \rightarrow P=\underbrace{\mathbf{X} \cdot \mathbf{S}}_{\mathbf{X}^{\prime}} \cdot \underbrace{\mathbf{S}^{-1} \cdot I}_{I^{\prime}}
$$

where $I^{\prime}$ is the new current vector in the new basis and $\mathbf{X}^{\prime}$ is

$$
\mathbf{X}^{\prime}=\left[\begin{array}{ccc}
V_{g}^{+} & 0 & 0 \\
0 & V_{g}^{+} & 0 \\
V_{g}^{-} \cos \psi & -V_{g}^{-} \sin \psi & -\frac{V_{g}^{+2}-V_{g}^{-2}}{\sqrt{V_{g}^{+2}+V_{g}^{-2}}}
\end{array}\right]
$$

When expressed in the new base, it is clear that a singular voltage condition makes the last column of (12) be zero. Under such condition, it is not possible to independently control $P_{1}$, $P_{2}$ and $P_{3}$ and a compromise solution is needed. One possible choice is to leave $P_{3}$ as a function of $P_{1}$ and $P_{2}$, under singular voltage conditions. This can be done by using the following modified inverse of matrix $\mathbf{X}^{\prime}$

$$
\left(\mathbf{X}^{\prime}\right)^{-1}=\left[\begin{array}{ccc}
\frac{1}{V_{g}^{+}} & 0 & 0 \\
0 & \frac{1}{V_{g}^{+}} & 0 \\
\beta C_{1}\left(\frac{V_{g}^{-} \cos \psi}{V_{g}^{+}}\right) & -\beta C_{1}\left(\frac{V_{g}^{-} \sin \psi}{V_{g}^{+}}\right) & -\beta C_{1}
\end{array}\right]
$$

where $\beta$ is a controllable weighting factor and

$$
C_{1} \triangleq \frac{\sqrt{V_{g}^{+2}+V_{g}^{-2}}}{V_{g}^{+2}-V_{g}^{-2}}
$$

The inverted matrix $\left(\mathbf{X}^{\prime}\right)^{-1}$ can be used under conditions where $V_{g}^{+}$is significantly different than $V_{g}^{-}$to obtain the same solution that would be obtained from (3). For conditions where $V_{g}^{+} \approx V_{g}^{-}, \beta$ can be set to zero to avoid large current reference values caused by the singularity in $C_{1}$. Finally, the desired current vector can be obtained in the original base by doing

$$
I=\left.\mathbf{S} \cdot\left(\mathbf{X}^{\prime}\right)^{-1}\right|_{\beta} \cdot P
$$

The elimination of one degree of freedom of the additive current in Method 2 makes $P_{3}$ be a function of $P_{1}$ and $P_{2}$ rather than tracking its reference value. $P_{3}$ is the sum of the power references $P_{l \rightarrow u}^{j}$ for the three phases in (1). Therefore, the controller will not be able to compensate the total deviation between upper and lower arms under singular voltage conditions until the AC network voltages are restored.

3) Method 3 - Pseudoinverse-based approach: The third method uses the Moore-Penrose pseudoinverse to find a different compromise solution to the incompatible system. Assuming that the third component of $I^{\prime}$ (see $\mathbf{X}^{\prime}$ in (12)) is made to be zero when $V_{g}^{+}=V_{g}^{-}$, the matrix $\mathbf{X}^{\prime}$ can be reduced to the following 3 by 2 matrix

$$
\mathbf{X}^{\prime \prime}=\left[\begin{array}{cc}
V_{g}^{+} & 0 \\
0 & V_{g}^{+} \\
V_{g}^{-} \cos \psi & -V_{g}^{-} \sin \psi
\end{array}\right]
$$

The Moore-Penrose pseudoinverse, can be used to find the current vector that leads to the feasible power exchange that is closest to the desired power. This idea has been extensively used in other fields such as in control of robot manipulators close to their singularities [17]. The pseudoinverse of $\mathbf{X}^{\prime \prime}$ (16) imposing the singular voltage condition is

$$
\left(\mathbf{X}^{\prime \prime}\right)^{-1}=\left[\begin{array}{ccc}
-\frac{\cos (2 \psi)-3}{4 V_{g}^{+}} & \frac{\sin (2 \psi)}{4 V_{g}^{+}} & \frac{\cos \psi}{2 V_{g}^{+}} \\
\frac{\sin (2 \psi)}{4 V_{g}^{+}} & \frac{\cos (2 \psi)+3}{4 V_{g}^{+}} & -\frac{\sin \psi}{2 V_{g}^{+}}
\end{array}\right]
$$

As with Method 2, the desired current vector can be obtained by undoing the kernel transformation with

$$
I=\mathbf{S} \cdot\left(\mathbf{X}^{\prime \prime}\right)^{-1} \cdot P
$$


4) Anti-windup implementation for the reference current: The proposed methods are able to maintain the additive current references bounded during singular voltage conditions. However, when the fault is cleared and normal operating conditions are recovered, integral windup problems may appear in the energy regulators that control the difference between the energy of the upper and the lower arms, $E_{l \rightarrow u}^{j}$. These problems are caused by the mismatch between the power set-points of the energy controllers $P_{l \rightarrow u}^{j *}$ and the actual power exchange. To overcome this problem, an antiwindup structure controlling the integral terms of the energy regulators must be implemented. This structure calculates the difference between the power reference given by the energy regulators $P_{l \rightarrow u}^{j *}$ and the actual achievable power exchange recalculated from the chosen current references. The error $P_{l \rightarrow u}^{j-w}$ is then subtracted to the input of the integrators to keep them from increasing indefinitely ${ }^{3}$.

5) Singular voltage condition and application of proposed alternative reference calculation methods: The AC additive reference calculation uses the grid voltage $V_{g}$. The positive $V_{g}^{+}$and negative $V_{g}^{-}$sequences of the grid voltage $V_{g}$ can be obtained from the Phase-Locked Loop (PLL). Whenever the difference in magnitude of the positive and the negative sequence voltages is below a defined threshold, one of the three proposed methodologies can be applied in order to avoid a large $\mathrm{AC}$ additive current reference value. The aforementioned method assumes that AC grid voltage is similar to the voltage applied by the converter considering that the impedances of the filters are small. Alternatively, the voltages applied by the converter could be estimated using the AC grid voltage, the filter impedance and the $\mathrm{AC}$ grid current references.

\section{ANALYSIS OF THE DIFFERENT TYPES OF VOLTAGES SAGS}

In this section, a detailed analysis of the specific voltage sags that can cause the singular voltage condition $\left(V_{g}^{+}=V_{g}^{-}\right)$ is performed. Table II shows a classification of the different types of voltage sags [18]. Note that while type A is a symmetrical voltage sag, the other types correspond to unbalanced cases. The types of voltage sags shown in Table II are caused by different types of faults [18]. Specifically, type A voltage sags are caused by three-phase faults, regardless of transformer winding and the type of connection. Type B voltage sags are only caused by Single-Line-to-Ground (SLG) faults. Types C and D are produced from either SLG or Line-to-Line (LL) faults and also, types E, F and $\mathrm{G}$ are only expected if the fault is Double-Line-to-Ground (LLG). The transformer winding and the type of connection varies the voltage sag type as the disturbance propagates through the power system.

Two variables are used to describe the three-phase voltages during the sag: the pre-fault voltage $E_{1}$ and the voltage in the faulted phase (or between faulted phases) $V$.

The analysis procedure consists of applying the Fortescue transformation [19] to the voltage equations for each type of

\footnotetext{
${ }^{3}$ Superscript $w$ in $P_{l \rightarrow u}^{j-w}$ is referenced to the anti-windup structure.
}

TABLE II

GENERAL CLASSIFICATION OF VOLTAGE SAGS

\begin{tabular}{|c|c|c|}
\hline Type & Voltages & Phasors $a b c$ \\
\hline A & $\begin{array}{c}\underline{V}_{g}^{a}=V \\
\underline{V}_{g}^{b}=-\frac{1}{2} V-\frac{1}{2} j V \sqrt{3} \\
\underline{V}_{g}^{c}=-\frac{1}{2} V+\frac{1}{2} j V \sqrt{3}\end{array}$ & \\
\hline B & $\begin{array}{c}\underline{V}_{g}^{a}=V \\
\underline{V}_{g}^{b}=-\frac{1}{2} E_{1}-\frac{1}{2} j E_{1} \sqrt{3} \\
\underline{V}^{c}=-\frac{1}{2} E_{1}+\frac{1}{2} j E_{1} \sqrt{3}\end{array}$ & \\
\hline $\mathrm{C}$ & $\begin{array}{c}\underline{V}_{g}^{a}=E_{1} \\
\underline{V}_{g}^{b}=-\frac{1}{2} E_{1}-\frac{1}{2} j V \sqrt{3} \\
\underline{V}_{g}^{c}=-\frac{1}{2} E_{1}+\frac{1}{2} j V \sqrt{3}\end{array}$ & \\
\hline $\mathrm{D}$ & $\begin{array}{c}\underline{V}_{g}^{a}=V \\
\underline{V}_{g}^{b}=-\frac{1}{2} V-\frac{1}{2} j E_{1} \sqrt{3} \\
\underline{V}_{g}^{c}=-\frac{1}{2} V+\frac{1}{2} j E_{1} \sqrt{3}\end{array}$ & \\
\hline $\mathrm{E}$ & $\begin{array}{c}\underline{V}_{g}^{a}=E_{1} \\
\underline{V}_{g}^{b}=-\frac{1}{2} V-\frac{1}{2} j V \sqrt{3} \\
\underline{V}_{g}^{c}=-\frac{1}{2} V+\frac{1}{2} j V \sqrt{3}\end{array}$ & \\
\hline $\mathrm{F}$ & $\begin{array}{c}\underline{V}_{g}^{a}=V \\
\underline{V}_{g}^{b}=-\frac{1}{2} V-\left(\frac{1}{3} E_{1}+\frac{1}{6} V\right) j \sqrt{3} \\
\underline{V}_{g}^{c}=-\frac{1}{2} V+\left(\frac{1}{3} E_{1}+\frac{1}{6} V\right) j \sqrt{3}\end{array}$ & \\
\hline G & $\begin{array}{c}\underline{V}_{g}^{a}=\frac{2}{3} E_{1}+\frac{1}{3} V \\
\underline{V}_{g}^{b}=-\frac{1}{3} E_{1}-\frac{1}{6} V-\frac{1}{2} j V \sqrt{3} \\
\underline{V}_{g}^{c}=-\frac{1}{3} E_{1}-\frac{1}{6} V+\frac{1}{2} j V \sqrt{3}\end{array}$ & \\
\hline
\end{tabular}

voltage sag. The Fortescue transformation $\underline{\Theta}^{+-0}$ of a phasor vector $\underline{\Theta}^{a b c}$ is defined as

$$
\underline{\Theta}^{+-0} \triangleq \mathbf{F} \cdot \underline{\Theta}^{a b c}=\frac{1}{3}\left[\begin{array}{ccc}
1 & \underline{p}^{2} & \frac{p}{p^{2}} \\
1 & \frac{p}{1} & \frac{p^{1}}{1}
\end{array}\right] \cdot \underline{\Theta}^{a b c}
$$

with $\underline{p}=e^{j \frac{-2 \pi}{3}}$. Then, the positive $\underline{V}_{g}^{+}$and negative $\underline{V}_{g}^{-}$ sequence phasors are made to be equal in magnitude in order to find the fault states $V$ that cause this condition. An example of this procedure is shown next for a voltage sag of type $\mathrm{C}$.

\section{A. Analysis of voltage sag type $C$}

The equations for the voltage sag type $\mathrm{C}$ are

$$
\underline{V}_{g}^{a b c}=\left[\begin{array}{c}
E_{1} \\
-\frac{1}{2} E_{1}-\frac{1}{2} j V \sqrt{3} \\
-\frac{1}{2} E_{1}+\frac{1}{2} j V \sqrt{3}
\end{array}\right]
$$

Applying the Fortescue transformation to (20) gives

$$
\underline{V}_{g}^{+-0}=\mathbf{F} \cdot \underline{V}_{g}^{a b c}=\left[\begin{array}{c}
\frac{1}{2} E_{1}+\frac{1}{2} V \\
\frac{1}{2} E_{1}-\frac{1}{2} V \\
0
\end{array}\right]
$$

Solving for the case when $\left|\underline{V}^{+}\right|=\left|\underline{V^{-}}\right|$yields

$$
\left|\frac{1}{2} E_{1}+\frac{1}{2} V\right|=\left|\frac{1}{2} E_{1}-\frac{1}{2} V\right|
$$


Assuming that $E_{1}$ and $V$ are positive, this equation implies that $V=0$. Therefore, the voltage in $a b c$ variables is of the form

$$
\underline{V}_{g}^{a b c}=\left.\mathbf{F}^{-1} \cdot \underline{V}_{g}^{+-0}\right|_{V=0}=\left[\begin{array}{c}
E_{1} \\
-\frac{1}{2} E_{1} \\
-\frac{1}{2} E_{1}
\end{array}\right]
$$

Following an analogous procedure for the other types of voltage sags, the results shown in Table III are obtained. Note that voltage sags type $\mathrm{A}^{4}$ and $\mathrm{B}$ cannot produce a singular voltage condition while types $\mathrm{C}, \mathrm{D}, \mathrm{E}, \mathrm{F}$ and $\mathrm{G}$ can.

TABLE III

VOLTAGE SAGS WITH SINGULAR VOLTAGE CONDITION

\begin{tabular}{|c|c|c|c|}
\hline Type & Voltages $a b c$ & Voltages +-0 & Phasors $a b c$ \\
\hline A & $\begin{array}{l}\underline{V}_{g}^{a}=0 \\
\underline{V}_{g}^{b}=0 \\
\underline{V}_{g}^{c}=0\end{array}$ & $\begin{array}{l}\underline{V}_{g}^{+}=0 \\
\underline{V}_{g}^{-}=0 \\
\underline{V}_{g}^{0}=0\end{array}$ & \\
\hline B & - & - & - \\
\hline $\mathrm{C}$ & $\begin{array}{c}\underline{V}_{g}^{a}=E_{1} \\
\underline{V}_{g}^{b}=-\frac{1}{2} E_{1} \\
\underline{V}_{g}^{c}=-\frac{1}{2} E_{1}\end{array}$ & $\begin{array}{c}\underline{V}_{g}^{+}=\frac{1}{2} E_{1} \\
\underline{V}_{g}^{-}=\frac{1}{2} E_{1} \\
\underline{V}_{g}^{0}=0\end{array}$ & \\
\hline D & $\begin{array}{c}\underline{V}_{g}^{a}=0 \\
\underline{V}_{g}^{b}=-\frac{1}{2} j E_{1} \sqrt{3} \\
\underline{V}_{g}^{c}=+\frac{1}{2} j E_{1} \sqrt{3}\end{array}$ & $\begin{array}{c}\underline{V}_{g}^{+}=\frac{1}{2} E_{1} \\
\underline{V}_{g}^{-}=-\frac{1}{2} E_{1} \\
\underline{V}_{g}^{0}=0\end{array}$ & \\
\hline $\mathrm{E}$ & $\begin{array}{c}\underline{V}_{g}^{a}=E_{1} \\
\underline{V}_{g}^{b}=0 \\
\underline{V}_{g}^{c}=0\end{array}$ & $\begin{array}{l}\underline{V}_{g}^{+}=\frac{1}{3} E_{1} \\
\underline{V}_{g}^{-}=\frac{1}{3} E_{1} \\
\underline{V}_{g}^{0}=\frac{1}{3} E_{1}\end{array}$ & \\
\hline $\mathrm{F}$ & $\begin{array}{c}\underline{V}_{g}^{a}=0 \\
\underline{V}_{g}^{b}=j \frac{\sqrt{3}}{3} E_{1} \\
\underline{V}_{g}^{c}=j \frac{\sqrt{3}}{3} E_{1}\end{array}$ & $\begin{array}{c}\underline{V}_{g}^{+}=\frac{1}{3} E_{1} \\
\underline{V}_{g}^{-}=-\frac{1}{3} E_{1} \\
\underline{V}_{g}^{0}=0\end{array}$ & \\
\hline G & $\begin{array}{c}\frac{V_{g}^{a}=\frac{2}{3} E_{1}}{V_{g}^{b}=-\frac{1}{3} E_{1}} \\
\underline{V}_{g}^{c}=-\frac{1}{3} E_{1}\end{array}$ & $\begin{array}{c}\underline{V}_{g}^{+}=\frac{1}{3} E_{1} \\
\underline{V}_{g}^{-}=\frac{1}{3} E_{1} \\
\underline{V}_{g}^{0}=0\end{array}$ & \\
\hline
\end{tabular}

\section{CASE STUDY}

In this section, different simulations are carried out in Matlab Simulink ${ }^{\circledR}$ to study the behavior of the proposed methods under the voltage conditions described in Table III. First, the results for the methodologies proposed are analyzed in detail for the voltage sag type C. Then, simulations are carried out for the rest of the voltage sags types focusing on the impact of each methodology in the converter energy. The system parameters used for this study are detailed in Table IV.

\section{A. Simulation model description}

The converter model implemented is based on the accelerated model proposed in [20]. This model allows to access each individual SM voltage providing enough accuracy compared

\footnotetext{
${ }^{4}$ For type A, the singular voltage condition appears when a short-circuit at the output of the converter is produced. Under such condition it is no possible to exchange any power with the AC grid, thus the upper-lower energy balancing control could be deactivated.
}

TABLE IV

SYSTEM PARAMETERS

\begin{tabular}{lccc}
\hline \hline Parameter & Symbol & Value & Units \\
\hline Rated power & $S$ & 526 & $\mathrm{MVA}$ \\
Rated power factor & $\cos \varphi$ & $0.95(\mathrm{c})$ & - \\
AC-side voltage & $U$ & 320 & $\mathrm{kV}$ rms ph-ph \\
HVDC link voltage & $V_{D C}$ & \pm 320 & $\mathrm{kV}$ \\
Grid equivalent impedance & $Z_{g}$ & $0.01+\mathrm{j} 0.1$ & $\mathrm{pu}$ \\
Phase reactor impedance & $Z_{s}$ & $\mathrm{j} 0.05$ & $\mathrm{pu}$ \\
Arm reactor impedance & $Z_{a}$ & $0.01+\mathrm{j} 0.2$ & $\mathrm{pu}$ \\
Converter modules per arm & $N_{\text {arm }}$ & 400 & 1 \\
Average module voltage & $V_{\text {module }}$ & 1.6 & $\mathrm{kV}$ \\
Sub-module capacitance & $C_{\text {module }}$ & 8 & $\mathrm{mF}$ \\
\hline \hline
\end{tabular}

to a fully-detailed model [21]. Each sub-module is represented by its equivalent capacitor that is charged and discharged depending on its insertion state. The modulation implemented is the Nearest Level Control (NLC) technique [22], which calculates the number of active sub-modules in each arm and uses a reduced switching strategy to increase the efficiency of the converter.

\section{B. Simulation results - Case $C$}

In this section, the behavior of the converter during a type $\mathrm{C}$ voltage sag with singular voltage condition is simulated. Four scenarios are considered: the first one is based on not taking any special precaution to handle the sag (Method 0) and it is shown in Fig. 5. The second one, based on Method 1 (disconnecting the energy balancing controller), is shown in Fig. 6. Method 2 (the kernel-based approach) is shown in Fig. 7. Finally, Method 3 (the pseudoinverse-based approach) can be seen in Fig. 8. A comparison between the four simulations is also shown in Fig. 9. The comparison is mainly done in terms of the evolution of the average energy difference between the upper and the lower arms of the converter during the fault, which is where the main differences between the aforementioned methods become apparent.

The simulated voltage sag starts at time $3 \mathrm{~s}$ and the voltage is fully restored at time $5 \mathrm{~s}$. Although in a real network a deep and highly unbalanced voltage sag condition would be sustained for less than $250 \mathrm{~ms}$ today [23], in this case, the sag has been extended for $2 \mathrm{~s}$, to highlight the differences between the different methods compared.

Also, Fig. 4 has been included as a detailed legend for Fig. 5, 6, 7 and Fig. 8 to clarify the waveforms that are being plotted in the different graphs. First, focusing on the AC side variables (1st column of Figs. 5, 6, 7 and 8), when the voltage sag appears it can be seen that the grid current control only injects positive sequence current to the grid, following the grid code requirements. No differences can be appreciated between the different methods, as the AC grid current control is completely decoupled from the upper-lower arm energy balancing problem. Also, the AC power exchanged with the grid has a double-line frequency component, as the voltage state is unbalanced and the converter is exchanging only positive sequence current.

Regarding the DC side variables (4th column of Figs. 5, 6, 7 and 8), the DC output current and power show the same 
response for the different methods. Analogously to the AC side control, the DC current control is independent from the problem of the upper-lower arm energy balancing, as it is related with the total energy stored in the converter. Note also that the double-line frequency power exchanged by the converter with the AC grid is not transmitted to the DC grid as in a two-level converter. This power oscillation remains within the converter.

In terms of the upper and lower arms variables (2nd and 3rd columns of Figs. 5, 6, 7 and 8), it can be seen that in the first milliseconds after the fault inception, the voltages, currents and power flows corresponding to the operation of the upper and lower arms are almost equivalent. Focusing on Method 0 , the converter currents are maintained under reasonable values as the output of the additive reference calculation is saturated to avoid sending large current references to the current controllers. The main difference between Method 0 and the proposed reference calculation modifications can be observed in Fig. 5b in the 4th row of the graph, where the total sum of the arm capacitors voltage of the upper and lower arms is depicted. Note that, this voltage magnitude is directly related with the energy stored in each of the arms, which will reflect the upper and lower arms energy balancing problem. Fig. $5 \mathrm{~b}$ shows that at $4 \mathrm{~s}$ the energy difference between phase $c$ upper and lower arms is increasing since the fault inception (as the sum voltage in the upper arm is still increasing and the sum voltage in the lower arm is decreasing), showing a noncontrolled energy deviation that leads to the disconnection of the converter. However, using Methods 1, 2 and 3 (Figs. 6b, $7 \mathrm{~b}$ and $8 \mathrm{~b}$ ) the average value of the arm capacitors voltage total sum is maintained constant during the fault.

In terms of the response after the fault is cleared, it can be seen that for Methods 1, 2 and 3 (see Figs. 6b, 7b and 8b) the AC grid, DC grid and converter arm variables show similar transients. All variables show an smooth recovery evolution to the pre-fault values.

In order to better understand the behavior of the differences between methods, Fig. 9 shows the average energy difference between the upper and lower arms of the MMC converter during the voltage sag. When Method 0 is applied (see Fig. 9a), although it has been seen that the converter variables appear to be stable during the first few cycles (see Fig. 5), a drift of the arm energies occurs leading to the converter disconnection around $1 \mathrm{~s}$ after the inception of the fault. In the simulation, the disconnection is triggered when there is a sustained deviation of the average arm energy of more than $5 \%$. The drift is caused by the saturation of the current references due to the singularity in the calculation of the additive AC current, which compromises the effectiveness of the energy regulators.

Also, Fig. 9b shows the evolution of the average energy difference between the upper and lower arms applying Method 1. It can be seen that the converter remains stable during the fault, even though the transient causes a deviation of the energy balance between upper and lower arms that is not corrected (see Fig. 6). When the fault is cleared, the energy deviation is compensated when the balance controller is enabled again (see Fig. 6b and Fig. 9b).

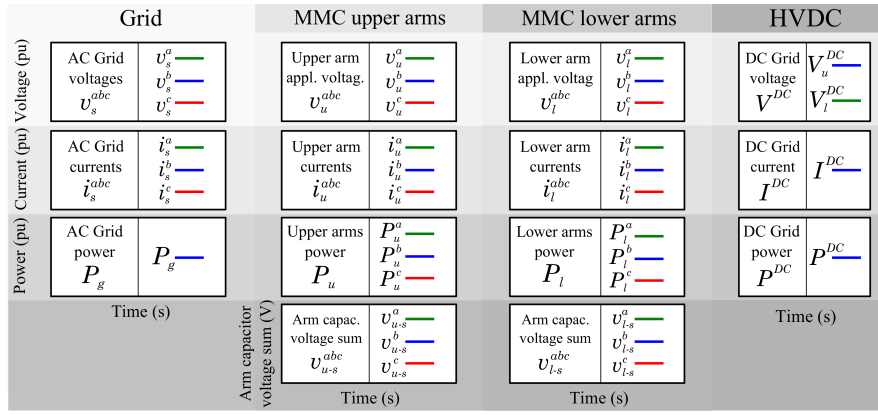

Fig. 4. Simulation results of the MMC operation. Graphs detailed legend.

The response can be improved by using Method 2 (see Fig. 7). With this method, a singular voltage condition does not cause the current references to saturate. Therefore, even though the deviation between upper and lower arms of the converter caused by the transient is not corrected during the voltage imbalance, the remaining energy balancing controls remain functional leading to a lower energy deviation (see Fig. 9c). It is worth noting that with this method the average energy imbalance between upper and lower arms during the fault is the same in each of the three legs of the converter. This is an inherent property of Method 2, which loses control over the total balance between upper and lower arms only. Also, the energy balance is fully recovered upon restoration of the network AC voltage (see Fig. 7b and Fig. 9c).

Finally, Fig. 9d shows the results for the last proposed solution, Method 3. The benefit of Method 3 when compared to Method 2 is that rather than dismissing $P_{3}$, it chooses the additive AC current reference that produces the closest feasible power to that requested by the energy balancing controls, which results in the lowest deviation of the energy balance, even though this may not be symmetrical when comparing the three legs of the converter (see Fig. 9d).

In addition, the different Methods show a different impact on the AC inner currents flowing through the converters. Fig. 10 shows the AC additive current reference output after the reference calculation scheme in the Clarke's reference frame $\alpha \beta 0$. Fig. 10a shows that Method 0 reaches the maximum AC allowed current reference when attempting to balance the upper and lower arms. Using this method, until the converter is disconnected, the reference calculation output remains at the maximum current level due to the division by zero. Fig. 10b does not show any impact over the AC currents as it consists on disconnecting the energy regulator that uses AC currents to balance the energy. Finally, Figs. 10c and 10d show the impact of Methods 2 and 3 over the AC additive current references, respectively. It can be observed that in both cases the system uses AC currents to balance the converter in a few milliseconds. After, when the converter reaches the steady state in both cases the current goes to zero, thus avoiding noneffective additive current flowing through the converter.

\section{Simulation results - Other cases}

Next, a series of simulations are performed for voltage sags type D, E, F and G with singular voltage condition. The 


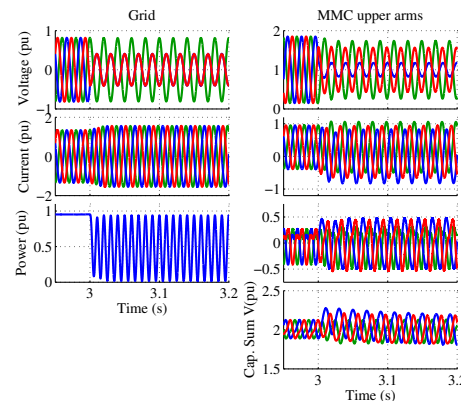

(a)
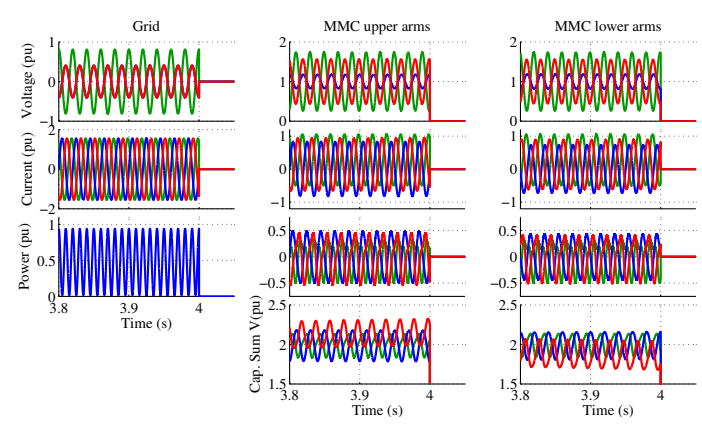

(b)

Fig. 5. Simulation results of the MMC operation. Voltage sag type C. Method 0 - Conventional control.

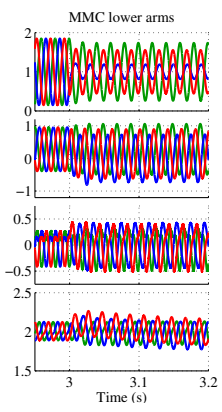

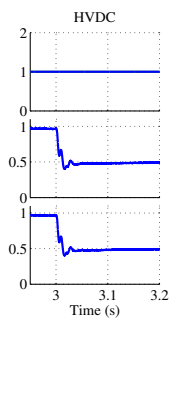
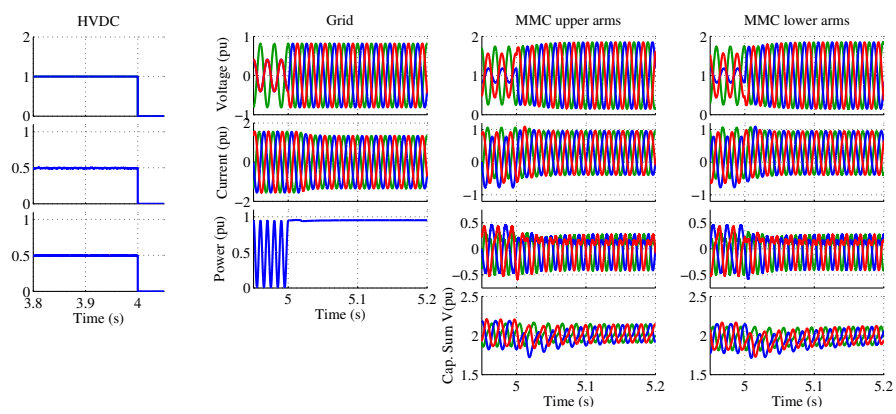

(b)

Fig. 7. Simulation results of the MMC operation. Voltage sag type C. Method 2 - Kernel-based approach.

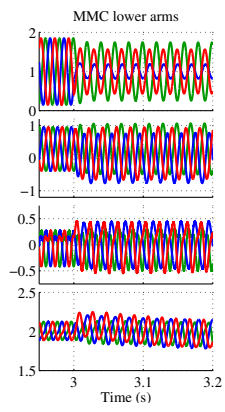

(a)

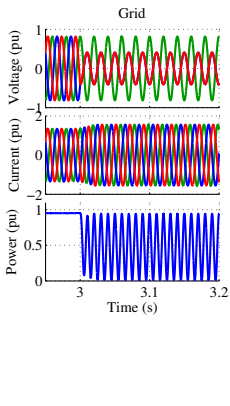

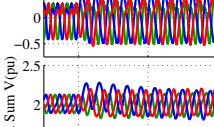
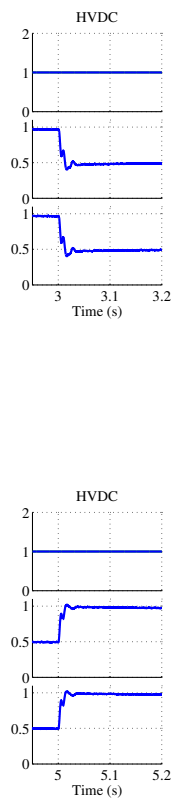

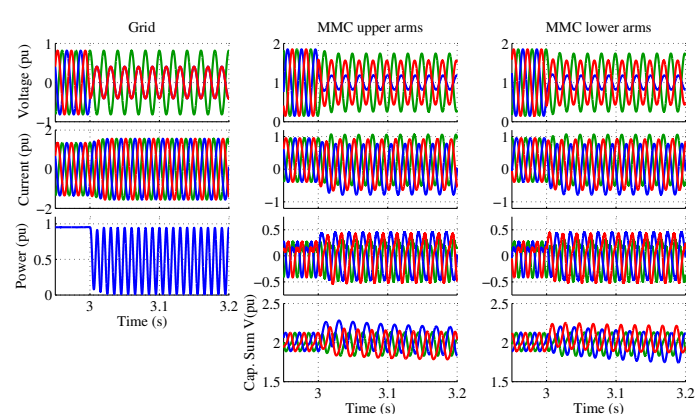

(a)

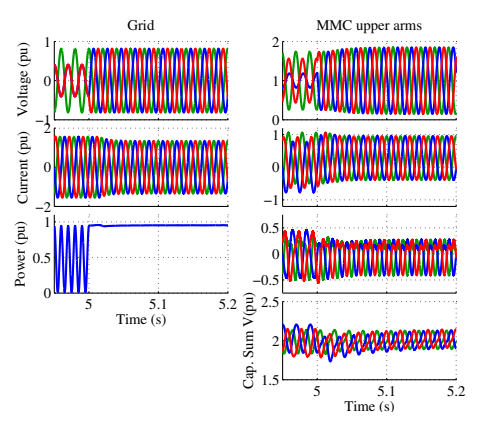

(b)
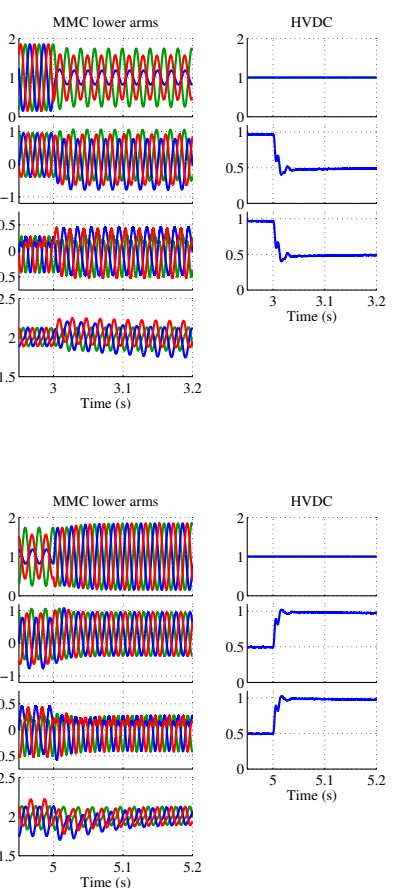

Fig. 6. Simulation results of the MMC operation. Voltage sag type C. Method 1 - Energy balancing disconnection.
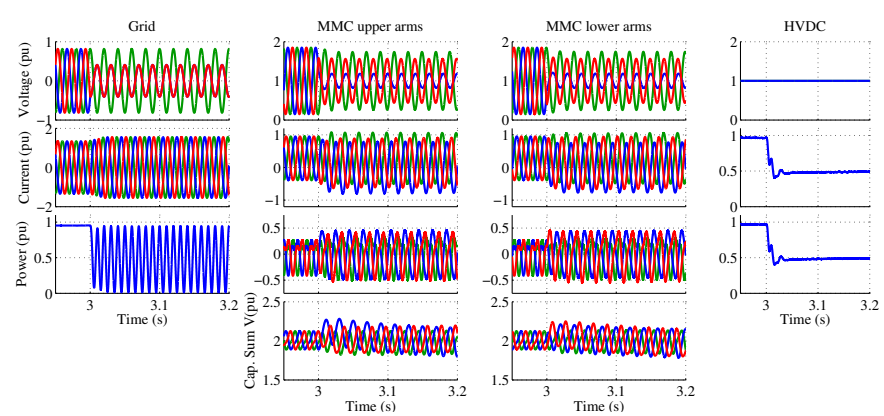

(a)
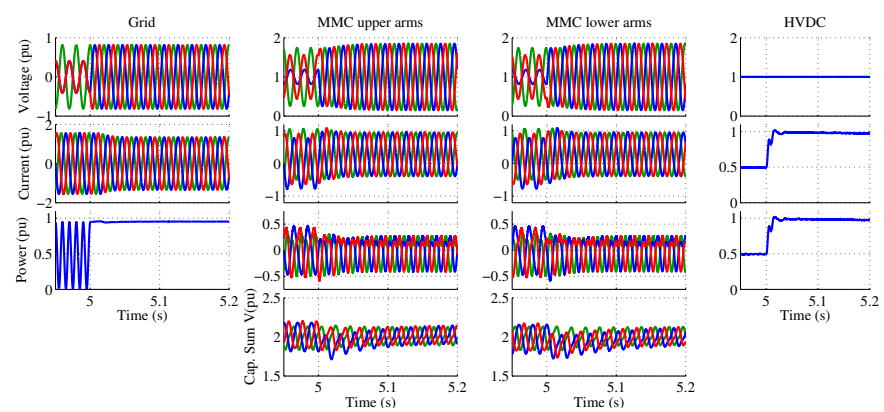

(b)

Fig. 8. Simulation results of the MMC operation. Voltage sag type C. Method 3 - Pseudoinverse-based approach. 

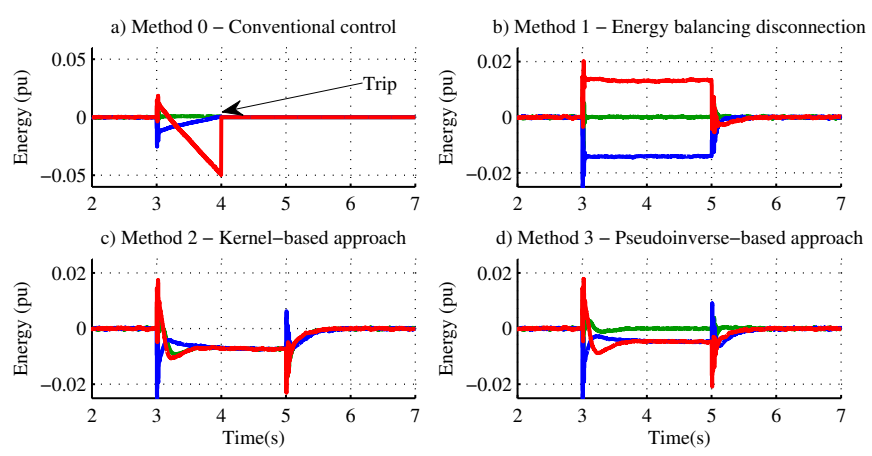

Fig. 9. Average energy comparison between the proposed methods. Voltage sag type C. Color code: $E_{l \rightarrow u}^{a}$ (green), $E_{l \rightarrow u}^{b}$ (blue), $E_{l \rightarrow u}^{c}$ (red).

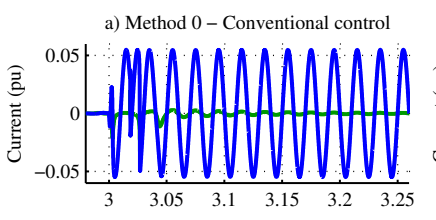

c) Method 2 - Kernel-based approach
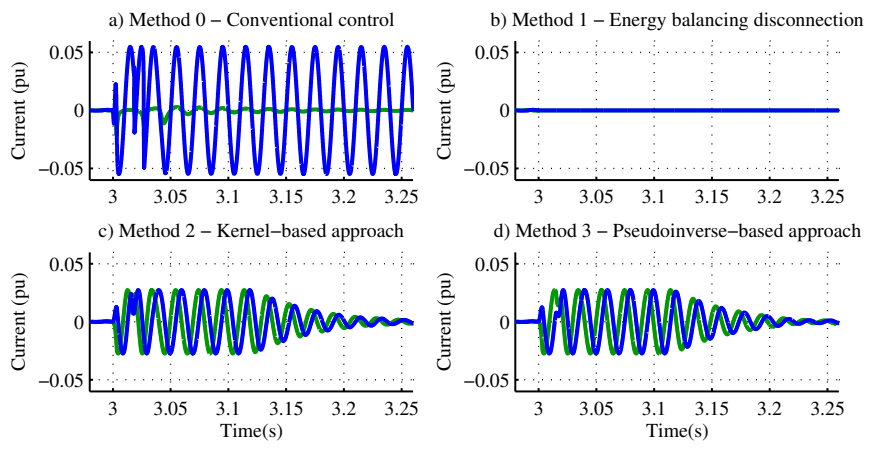

Fig. 10. AC additive current references obtained at the output of the reference calculation stage. Voltage sag type C. Color code: $i_{\text {sum }}^{\alpha *}$ (green), $i_{\text {sum }}^{\beta *}$ (blue).

purpose of these tests is to confirm the conclusions obtained for a type $\mathrm{C}$ voltage sag can also be extended to all other sags. The results are focused on the evolution of the average energy balance between the upper and the lower arms and are shown in Fig. 11 for type D, Fig. 12 for type E, Fig. 13 for type F and Fig. 14 for type G.

The simulation results confirm that Method 0 results in a sustained energy drift that eventually leads to the disconnection of the converter. In contrast, Methods 1, 2 and 3 keep the energy balance stable. Method 1 results in greater deviation of the energy and must be disabled upon recovery of normal voltage conditions while Methods 2 and 3 present a smooth transition between fault and normal operating conditions and result in lower energy deviation. Method 2 produces the same imbalance between the energy of the upper and the lower arms in all three legs of the converter while Method 3 results in an asymmetrical imbalance with lower total deviation.

In terms of the dynamic response (see Figs. 9-14), Method 1 shows a fast evolution to the steady state value as no control is considered and the deviation is mainly caused by a transient power disturbance. On the other hand, Methods 2 and 3 show a controlled evolution towards the steady state due to the energy regulator action. It should be mentioned a different control design may improve the system response allowing to reach the steady state faster.

\section{Differences between reference calculation methods}

In this section, the main differences between proposed methods are highlighted based on the previous results in terms of control, implementation, steady state error and current requirements. The main difference between Method 1 and Methods 2 and 3 is the action of the energy regulation controller. For Method 1, the energy controller is deactivated thus not sending AC power references, whereas for Methods 2 and 3 , the power outputs extracted from the regulator are fed into the reference calculation stage. Thus, it can be stated that Methods 2 and 3 are actively balancing the energy deviation between the upper and lower arms, while Method 1 is not performing an active control.

Regarding the implementation on a real system controller, the proposed methods show different degree of complexity from a computational perspective. The implementation of Method 1 is extremely simple, as it consists on deactivating the energy regulator. To implement Method 2 the complexity is increased as the normal reference calculation (Method 0) has to be adapted including the parameter $\beta$ in the system of equations resolution. However, Method 3 is the most resource time consuming methodology as it needs to calculate the Moore-penrose pseudoinverse to obtain the adequate current references.

From the point of view of the average energy deviation during the sag, Method 1 consists on deactivating the energy regulation, driving the system to a larger energy deviation compared to Methods 2 and 3. Method 2 is able to balance the energy difference between the upper and lower arms of the three phases to the same value. However, it is not able to compensate the deviation offset. Regarding Method 3, it reduces the error in all phases, so that the total converter energy deviation is smaller.

Another important difference to be studied is the amount of AC additive current that each method needs to balance the converter energies. Method 1 does not require any $\mathrm{AC}$ additive current, thus avoiding any possible overload that may appear during transients. On the other hand, Methods 2 and 3 use $\mathrm{AC}$ additive current to balance the converter energies during the sag (see Fig. 10). Both methods require approximately the same amount of AC additive current during a voltage sag to balance the converter only during a few milliseconds, which does not cause an important impact on the converter additive currents. Note that the additive currents, can be understood as current flowing through the upper and lower converter arms.

In summary, it can be seen that Method 1 is straightforwardly implementable, it does not have an impact in the converter currents during the sag, but it shows a larger energy deviation. Regarding Method 2, it does not importantly increase the implementation complexity compared to the normal calculation (Method 0), it is able to drive the converter to an equivalent energy balanced state per phase, flowing a certain amount of AC additive current through the converter arms. Finally, Method 3 is able to achieve a reduced energy deviation state using AC additive current compared to Methods 1 and 3. However, this technique requires a more complex reference calculation strategy based on the Moore-penrose pseudoin- 
verse. To conclude, the methodology to be implemented must be selected based on the converter controller specifications and also on the system requirements during operation.
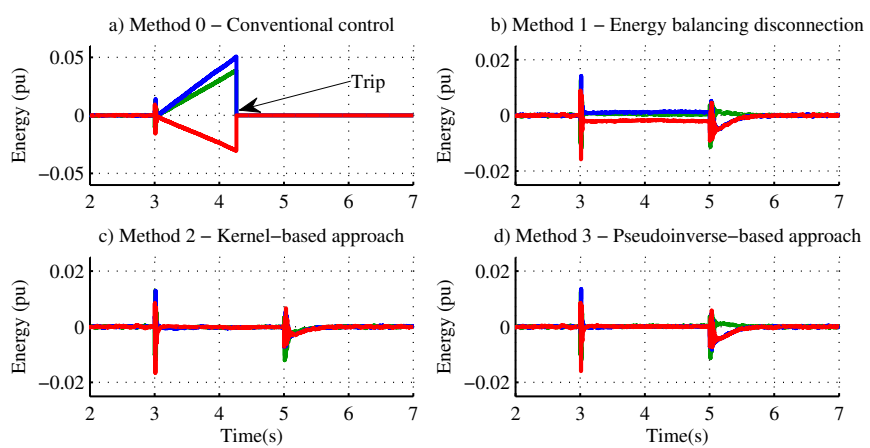

d) Method 3 - Pseudoinverse-based approach

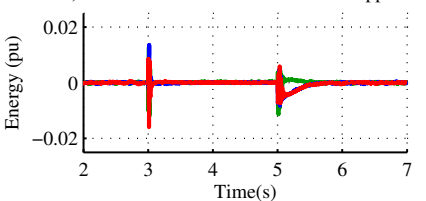

Fig. 11. Average energy comparison between the proposed methods. Voltage sag type D. Color code: $E_{l \rightarrow u}^{a}$ (green), $E_{l \rightarrow u}^{b}$ (blue), $E_{l \rightarrow u}^{c}$ (red).

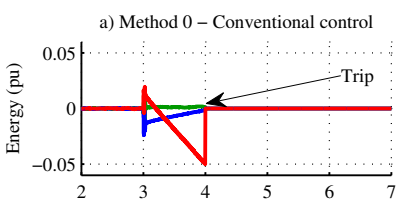

c) Method 2 - Kernel-based approach
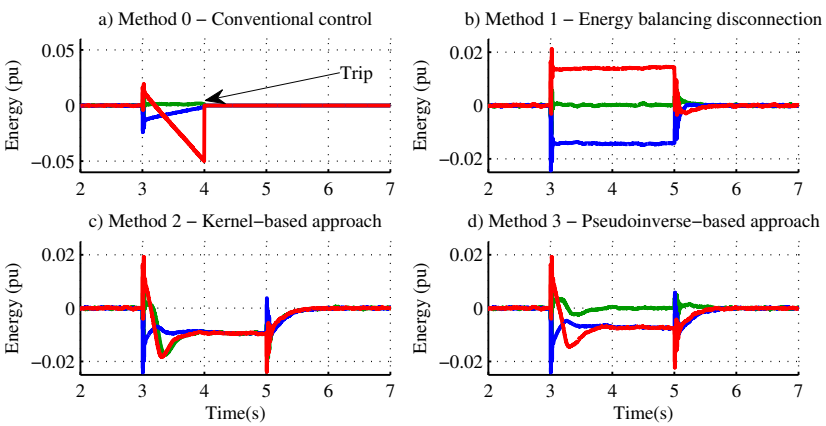

d) Method 3 - Pseudoinverse-based approach

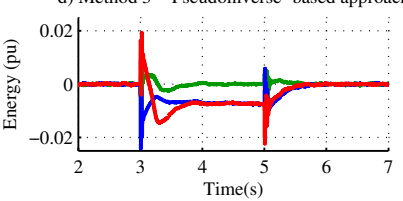

Fig. 12. Average energy comparison between the proposed methods. Voltage sag type E. Color code: $E_{l \rightarrow u}^{a}$ (green), $E_{l \rightarrow u}^{b}$ (blue), $E_{l \rightarrow u}^{c}$ (red).

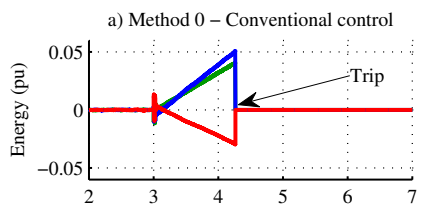

b) Method 1 - Energy balancing disconnection
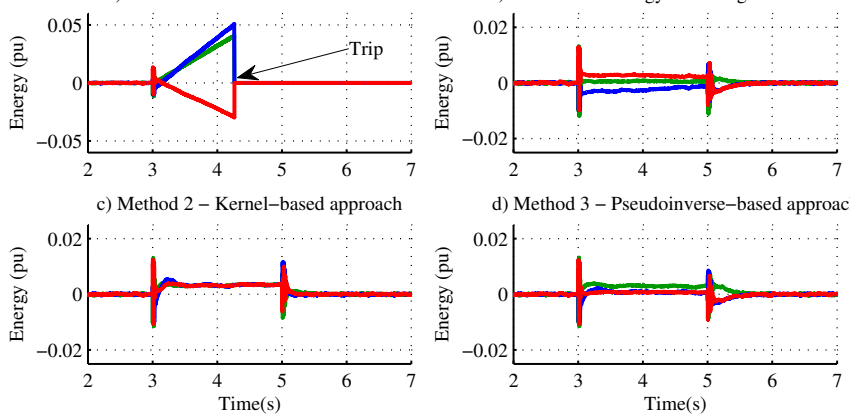

Fig. 13. Average energy comparison between the proposed methods. Voltage sag type F. Color code: $E_{l \rightarrow u}^{a}$ (green), $E_{l \rightarrow u}^{b}$ (blue), $E_{l \rightarrow u}^{c}$ (red).

\section{CONCLUSIONS}

Unbalanced voltage sags with singular voltage condition pose a challenge for the regulation of the energy balance between the upper and the lower arms of the converter. The analysis presented in this paper has shown how this specific condition may cause a singularity in the conventional current reference calculation. This singularity causes the current references to saturate, which compromises the performance of the
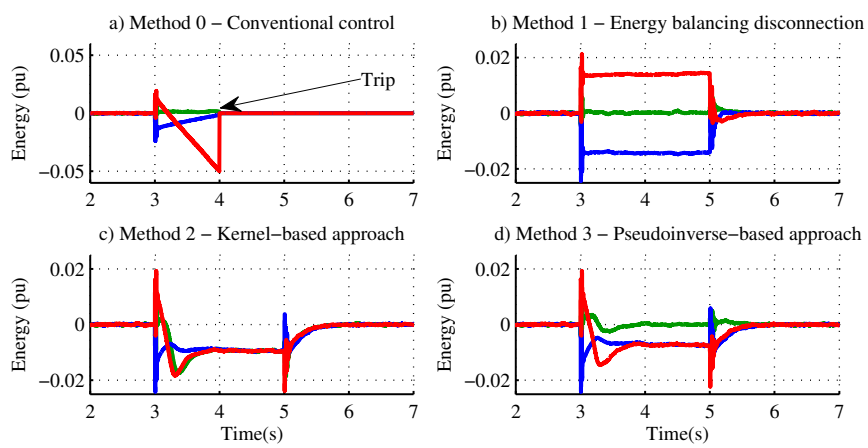

Fig. 14. Average energy comparison between the proposed methods. Voltage sag type G. Color code: $E_{l \rightarrow u}^{a}$ (green), $E_{l \rightarrow u}^{b}$ (blue), $E_{l \rightarrow u}^{c}$ (red).

energy balancing controllers. In order to overcome this issue, one option is to disable the energy balancing controller only during the fault, which makes the system stable but results in greater energy deviation. Alternatively, two new calculation methods have been presented which improve the deviation of the energy during the fault, regaining normal operation once the fault is cleared. The bases of the aforementioned methods have been discussed in detail and their benefits have been validated in a simulation model under all possible AC fault scenarios that produce the singular voltage condition.

\section{REFERENCES}

[1] O. Gomis-Bellmunt, J. Liang, J. Ekanayake, R. King, and N. Jenkins, "Topologies of multiterminal HVDC-VSC transmission for large offshore wind farms," Electric Power Syst. Res., vol. 81, no. 2, pp. 271-281, 2011.

[2] P. Wang, X.-P. Zhang, P. Coventry, and R. Zhang, "Start-up control of an offshore integrated mmc multi-terminal hvdc system with reduced dc voltage," IEEE Trans. Power Syst., vol. PP, no. 99, pp. 1-12, 2015.

[3] A. Lesnicar and R. Marquardt, "An innovative modular multilevel converter topology suitable for a wide power range," in IEEE Power Tech Conf. Proc, 2003, Bologna, vol. 3, June 2003, pp. 6 pp. Vol.3-.

[4] J. Dorn, H. Huang, and D. Retzmann, "A new multilevel voltagesourced converter topology for hvdc applications," CIGRE Session. Paris, France: Int. Council on Large Electric Syst., pp. 1-8, 2008.

[5] B. Jacobson, P. Karlsson, G. Asplund, L. Harnefors, and T. Jonsson, "Vsc-hvdc transmission with cascaded two-level converters," in Cigré session, 2010, pp. B4-B110.

[6] M. Merlin, T. Green, P. Mitcheson, D. Trainer, D. Critchley, and R. Crookes, "A new hybrid multi-level voltage-source converter with dc fault blocking capability," in 9th IET Int. Conf. on AC and DC Power Transmission, 2010. ACDC., Oct 2010, pp. 1-5.

[7] A. Antonopoulos, L. Angquist, and H.-P. Nee, "On dynamics and voltage control of the modular multilevel converter," in 13th European Conf. on Power Electronics and Applicat., 2009. EPE '09., Sept 2009, pp. 1-10.

[8] Q. Tu, Z. Xu, and L. Xu, "Reduced switching-frequency modulation and circulating current suppression for modular multilevel converters," IEEE Trans. Power Del., vol. 26, no. 3, pp. 2009-2017, July 2011.

[9] L. Harnefors, A. Antonopoulos, S. Norrga, L. Angquist, and H.-P. Nee, "Dynamic analysis of modular multilevel converters," IEEE Trans. Ind. Electron., vol. 60, no. 7, pp. 2526-2537, July 2013.

[10] M. Hagiwara, R. Maeda, and H. Akagi, "Control and analysis of the modular multilevel cascade converter based on double-star chopper-cells (mmcc-dscc)," IEEE Trans. Power Electron., vol. 26, no. 6, pp. 16491658 , June 2011.

[11] A. Antonopoulos, L. Angquist, L. Harnefors, K. Ilves, and H.-P. Nee, "Global asymptotic stability of modular multilevel converters," IEEE Trans. Ind. Electron., vol. 61, no. 2, pp. 603-612, Feb 2014.

[12] A. Junyent-Ferre, P. Clemow, M. Merlin, and T. Green, "Operation of hvdc modular multilevel converters under dc pole imbalances," in 16th European Conf. on Power Electron. and Applicat. (EPE'14-ECCE Europe), 2014, Aug 2014, pp. 1-10. 
[13] J.-J. Jung, S. Cui, S. Kim, and S.-K. Sul, "A cell capacitor energy balancing control of modular multilevel converter considering the unbalanced AC grid conditions," in Int. Power Electron. Conf. (IPEC), Hiroshima, Japan, May 2014, pp. 1268-1275.

[14] G. Tang, Z. Xu, and Y. Zhou, "Impacts of three mmc-hvdc configurations on ac system stability under dc line faults," IEEE Trans. Power Syst., vol. 29, no. 6, pp. 3030-3040, Nov 2014.

[15] A. Junyent-Ferre, O. Gomis-Bellmunt, T. Green, and D. Soto-Sanchez, "Current control reference calculation issues for the operation of renewable source grid interface vscs under unbalanced voltage sags," IEEE Trans. Power Electron., vol. 26, no. 12, pp. 3744-3753, Dec 2011.

[16] J.-J. Jung, S. Cui, Y. Lee, and S.-K. Sul, "A cell capacitor energy balancing control of MMC-HVDC under the AC grid faults," in 9th Int. Conf. on Power Electron. and ECCE Asia (ICPE-ECCE Asia), Seoul, South Korea, Jun. 2015, pp. 1-8.

[17] B. Siciliano, L. Sciavicco, L. Villani, and G. Oriolo, Robotics: Modelling, Planning and Control, ser. Advanced Textbooks in Control and Signal Processing. Springer London, 2010

[18] M. Bollen and L. Zhang, "Different methods for classification of threephase unbalanced voltage dips due to faults," Electric Power Syst. Res., vol. 66, no. 1, pp. 59 - 69, 2003, power Quality.

[19] C. L. Fortescue, "Method of symmetrical co-ordinates applied to the solution of polyphase networks," Trans. of the Amer. Institute of Elect. Engineers, no. 2, pp. 1027-1140, 1918.

[20] J. Xu, C. Zhao, W. Liu, and C. Guo, "Accelerated model of modular multilevel converters in pscad/emtdc," IEEE Trans. Power Del., vol. 28 , no. 1, pp. 129-136, Jan 2013.

[21] A. Beddard, M. Barnes, and R. Preece, "Comparison of detailed modeling techniques for mmc employed on vsc-hvdc schemes," IEEE Trans. Power Del., vol. 30, no. 2, pp. 579-589, April 2015.

[22] Q. Tu and Z. Xu, "Impact of sampling frequency on harmonic distortion for modular multilevel converter," IEEE Trans. Power Del., vol. 26, no. 1, pp. 298-306, Jan 2011.

[23] ENTSO-E, "Entso-E network code for requirements for grid connection applicable to all generators," Available at: https://www.entsoe.eu [accessed on February 2016]., 2013.

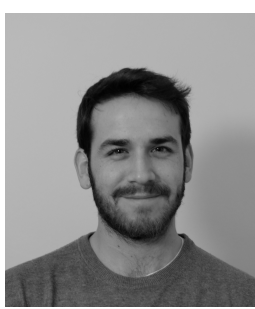

Eduardo Prieto-Araujo (S'12-M'16) received the degree in Industrial Engineering from the School of Industrial Engineering of Barcelona (ETSEIB), Technical University of Catalonia (UPC), Barcelona, Spain, in 2011, and the Ph.D. degree in Electrical Engineering from the UPC in 2016. Since 2010, he has been with the Centre d'Innovació Tecnològica en Convertidors Estàtics i Accionaments, (CITCEAUPC). His research interests include the modeling and control of electrical machines, renewable genelectronic converters for HVDC transmission.

eration systems, microgrids and control of power

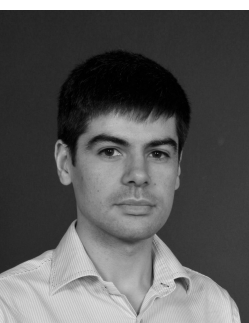

Adrià Junyent-Ferré (S'09-M'11) received the degree in Industrial Engineering from the School of Industrial Engineering of Barcelona (ETSEIB), Technical University of Catalonia (UPC), Barcelona, Spain, in 2007, and the Ph.D. degree in Electrical Engineering from the UPC in 2011. He was a researcher at Centre d'Innovació Tecnològica en Convertidors Estàtics i Accionaments (CITCEA-UPC) from 2006 to 2012 and a Lecturer at Barcelona College of Industrial Engineering (EUETIB) in 2012. He joined the Department of Electrical and Electronic Engineering of Imperial College London as a Research Associate in 2013 and he was appointed as a Lecturer in HVDC power transmission in September 2014. His research focusses on control of power electronic converters for large scale wind power generation and HVDC power transmission.

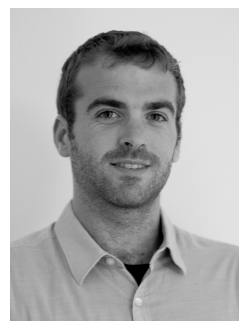

Gerard Clariana-Colet received the degree in Industrial Engineering from the School of Industrial Engineering of Barcelona (ETSEIB), Technical University of Catalonia (UPC), Barcelona, Spain. He was with the Centre d'Innovació Tecnològica en Convertidors Estàtics i Accionaments, (CITCEAUPC) from 2012 to 2015 where he worked with microgrids control and control of power electronic converters for HVDC grids. Since October 2015, he is with Ing. Karl und Albert KRUCH GmbH (KRUCH) working on the optimitzation of the energy flow for the railway DC networks.

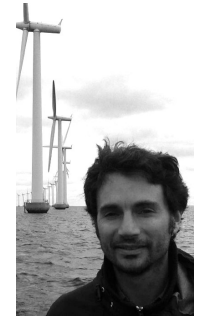

Oriol Gomis-Bellmunt (S'05-M'07-SM'12) received the degree in industrial engineering from the School of Industrial Engineering of Barcelona (ETSEIB), Technical University of Catalonia (UPC), Barcelona, Spain, in 2001 and the $\mathrm{PhD}$ in electrical engineering from the UPC in 2007. In 1999 he joined Engitrol S.L. where he worked as project engineer in the automation and control industry. In 2003 he developed part of his $\mathrm{PhD}$ thesis in the DLR (German Aerospace center) in Braunschweig (Germany). Since 2004 he is with the Electrical Engineering Department of the UPC where he is lecturer and participates in the CITCEA-UPC research group. Since 2009 he is also with the Catalonia Institute for Energy Research (IREC). His research interests include the fields linked with smart actuators, electrical machines, power electronics, renewable energy integration in power systems, industrial automation and engineering education. 